\title{
Sur l'analyse modale expérimentale des structures non-linéaires
}

\author{
Imad Tawfiq a ${ }^{\mathrm{a}}$, Tuong Vinh et Jean-Baptiste CasimiR \\ ISMCM, LISMMA - Rhéologie et Structures, 3 rue F. Hainaut, 93407 Saint-Ouen, France
}

Reçu le 6 juin 2003, accepté le 3 septembre 2004

\begin{abstract}
Résumé - Sur le plan expérimental, l'analyse modale des structures non-linéaires est encore en butte à de nombreuses difficultés. Nous voulons montrer que l'utilisation exclusive de la transformée de Fourier unidimensionnelle ne permet pas d'obtenir les vraies fonctions de transfert nécessaires pour atteindre les fonctionnelles non-linéaires de Volterra. L'analyse jusqu'ici se cantonne dans la majorité des cas à l'obtention des valeurs propres de divers modes non-linéaires. Il est nécessaire d'obtenir par un moyen ou par un autre, les noyaux (ou réponses impulsionnelles) des fonctionnelles permettant d'évaluer les vecteurs propres correspondants. Nous utilisons la technique de multi-excitation pour déterminer les fonctionnelles non-linéaires.
\end{abstract}

Mots clés : Analyse modale / non-linéarité / série de Volterra / réponses impulsionnelles / réponses fréquentielles / polyréférence

\begin{abstract}
About the experimental modal analysis of non-linear structures. Extensive research in experimental modal analysis of non-linear structures during these last twenty years are focussed on evaluation of eigenvalues of non-linear modes. Attempts must be made to reach true transfer functions which are the multidimensional Fourier transforms of Volterra functional kernels of the same order. Degenerated transfer functions or frequency responses in the majority of cases are often obtained by one-dimensional Fourier transforms. Consequently Volterra kernels cannot be evaluated by this way and generalized superposition principles included in the Volterra series are not applicable. In this paper we suggest some methods to evaluate these kernels. We used the polyreference technique to determine the non-linear functionals.
\end{abstract}

Key words: Modal analysis / non-linearity / Volterra series / impulse responses / frequency responses / polyreference

\section{Introduction}

En dépit de recherches actives, l'analyse modale nonlinéaire, est encore au stade de ses balbutiements. On est en mesure de décomposer les composantes linéaires et non-linéaires des réponses d'une structure pour obtenir, pour chaque mode non-linéaire, des valeurs propres. On utilise en pratique les transformées de Fourier unidimensionnelles pour obtenir les fonctions de transfert dégénérées encore appelées : réponses fréquentielles.

Rappelons que, dans la définition de la série de Volterra, les transformées directes de Fourier à $n$ dimensions des noyaux des fonctionnelles non linéaires d'ordre $n$ sont les fonctions de transfert correspondantes [1].

Dans la première partie nous présentons quelques méthodes pour évaluer les noyaux de ces fonctionnelles et, compte tenu des relations liant les vraies fonctions

\footnotetext{
a Auteur correspondant : tawfig@ismcm-cesti.fr
}

de transfert et les réponses fréquentielles, nous pourrons exploiter les résultats expérimentaux existants pour compléter les informations sur ces noyaux.

Ensuite nous présenterons l'étude d'une maquette de support moteur utilisé en construction navale (berceau) constitué de poutres cylindriques et de plaques assemblées par goupilles. Ces dernières assemblées en force et très proches les unes des autres, sont le siège de non-linéarités dues aux frottements secs et au serrage. Ainsi le comportement non-linéaire doit être pris en compte.

Une analyse modale classique préliminaire a montré la présence des modes de vibrations non-linéaires. Il est nécessaire d'envisager une analyse non-linéaire qui dépasse le cadre de l'analyse modale classique.

La technique de la polyréférence (c'est-à-dire la multiexcitation simultanée) existe déjà en analyse linéaire. Toutefois compte tenu des non-linéarités de la structure, il serait intéressant de voir quel sera le cadre théorique adéquat en vue de l'extension de l'analyse modale aux 


$$
\Lambda_{n}\left(f_{1}, \ldots, f_{n}\right)=\frac{\int_{-\infty}^{+\infty} \cdots \int_{-\infty}^{+\infty} H_{n}\left(f_{1}, f_{2}-f_{1}, f_{3}-f_{2}, \ldots, f-f_{n-1}\right) X\left(f_{1}\right) X\left(f_{2}-f_{1}\right) \ldots X\left(f-f_{n-1}\right) \mathrm{d} f_{1} \ldots \mathrm{d} f_{n-1}}{X\left(f_{1}\right) X\left(f_{2}\right) \ldots X\left(f_{n}\right)}
$$

structures non-linéaires. À cet égard nous présentons succinctement l'extension de la série de Volterra aux structures à entrées multiples et à sorties multiples. Dans une publication récente, Tawfiq et al. [2], nous avons présenté le cadre théorique qui permettait une telle extension.

Sur le plan expérimental l'utilisation de divers modes d'excitation a été utilisée : mono-excitation en bruit blanc, Gifford [3], mono-excitation sinusoïdale, Storer [4], excitation par chocs en un point ou en plusieurs points simultanés, Tawfiq [5].

À notre connaissance, seules les excitations par chocs ont permis de calculer les noyaux des fonctionnelles de Volterra. Ainsi est-il permis d'envisager l'analyse modale non-linéaire par la généralisation du principe de superposition jusqu'ici cantonné aux systèmes non-linéaires.

La structure mécanique non-linéaire considérée comme ayant des entrées et des sorties multiples n'est qu'une extension de l'analyse modale linéaire classique.

Il est intéressant de noter toutefois que l'interaction des excitations multiples varie selon les non-linéarités locales ou réparties. Leur importance diffère d'un endroit à l'autre et certaines d'entre elles n'ont aucune action sur les réponses observées assez loin des excitations.

S'agissant de l'analyse harmonique des réponses des structures non-linéaires, on peut dire que les harmoniques, les sous-harmoniques des fréquences primaires peuvent être détectées.

Par ailleurs, il existe des fréquences dites fractionnaires qui sont combinaisons linéaires des fréquences primaires telles que

$$
p f=\sum_{i=1}^{n} a_{i} f_{i}
$$

$a_{i}$ sont des nombres entiers positifs ou négatifs, $p$ un entier positif, $f_{i}$ les fréquences primaires, et $f$ la fréquence détectée.

\section{Séries de Volterra et transformées de Fourier}

\subsection{Réponses fréquentielles}

Rappelons la définition de cette série, $x(t)$ étant l'excitation et $y(t)$ la réponse de la structure, $y_{0}$ la composante continue :

$$
\begin{array}{r}
y(t)=y_{0}+\sum_{n=1}^{\infty} \int_{-\infty}^{+\infty} \cdots \int_{-\infty}^{+\infty} h_{n}\left(\tau_{1}, \ldots, \tau_{n}\right) \prod_{i=1}^{n} \\
\times x\left(t-\tau_{i}\right) \mathrm{d} \tau_{i}
\end{array}
$$

$n$ fois

$$
y(t)=y_{0}+\sum_{n=1}^{\infty} y_{n}(t)
$$

$h_{n}\left(\tau_{1}, \ldots, \tau_{n}\right)$ le noyau de la fonctionnelle d'ordre $n$ est une fonction à $n$ variables temporelles.

La transformée directe de Fourier à $n$ dimensions de temps permet d'obtenir la fonction de transfert, $f_{i}$ la fréquence, on désigne par lettres majuscules les transformées de Fourier des fonctions temporelles :

$$
\begin{aligned}
H_{n}\left(f_{1}, \ldots, f_{n}\right) & =\int_{-\infty}^{+\infty} \ldots \int_{-\infty}^{+\infty} h_{n}\left(t_{1}, \ldots, t_{n}\right) \\
& \times \exp \left[-2 j \pi\left(f_{1} t_{1}+\ldots+f_{n} t_{n}\right)\right] \mathrm{d} t_{1} \ldots \mathrm{d} t_{n}
\end{aligned}
$$

$n$ fois.

En utilisant la transformée de Fourier à une dimension on obtient en tronquant à l'ordre $n$ :

$$
Y(f)=Y_{1}(f)+Y_{2}(f)+\ldots+Y_{n}(f)
$$

On peut montrer, [5], que la composante $Y_{n}(f)$ s'écrit en utilisant (1)

$$
\begin{aligned}
& Y_{n}(f)=\int_{-\infty}^{+\infty} \ldots \int_{-\infty}^{+\infty} H_{n}\left(f_{1}, f_{2}-f_{1}, f_{3}-f_{2}, \ldots, f-f_{n-1}\right) \\
& \quad \times X\left(f_{1}\right) X\left(f_{2}-f_{1}\right) \ldots X\left(f-f_{n-1}\right) \mathrm{d} f_{1} \ldots \mathrm{d} f_{n-1}
\end{aligned}
$$

$(n-1)$ fois.

On définit la composante d'ordre $n$ de la réponse par :

$$
Y_{n}(f)=\Lambda_{n}\left(f_{1}, \ldots, f_{n}\right) X\left(f_{1}\right) X\left(f_{2}\right) \ldots X\left(f_{n}\right)
$$

et en portant l'équation (5) dans l'équation (4) on obtient la relation entre la fonction de transfert $H_{n}(f)$ et $\Lambda_{n}(f)$ avec $f=f_{1}+f_{2}+\ldots+f_{n}$

$$
\text { voir équation (6) au-dessus }
$$

La multiconvolution au second membre de l'équation (6) montre que la connaissance de $\Lambda_{n}(f)$ ne permet pas d'accéder à $H_{n}\left(f_{1}, \ldots, f_{n}\right)$.

\subsubsection{Excitation en bruit blanc}

L'image de Fourier de l'excitation $x(t)$ est une constante

$$
X(f)=a
$$

Portant l'équation (7) dans (6) on obtient

$$
\begin{array}{r}
\Lambda_{n}\left(f_{1}, \ldots, f_{n}\right)=\int_{-\infty}^{+\infty} \ldots \int_{-\infty}^{+\infty} H_{n}\left(f_{1}, f_{2}-f_{1}, \ldots,\right. \\
\left.f_{n-1}-f_{n-2}, f_{n}-f_{n-1}\right) \mathrm{d} f_{1} \ldots \mathrm{d} f_{n-1}
\end{array}
$$




$$
\Lambda_{n}\left(f_{1}, \ldots, f_{n}\right)=\frac{\int_{-\infty}^{+\infty} \ldots \int_{-\infty}^{+\infty} H_{n}\left(f_{1}, f_{2}-f_{1}, \ldots, f-f_{n-1}\right) \delta\left(f_{1}-f_{a}\right) \delta\left(f_{2}-f_{1}-f_{a}\right) \ldots \delta\left(f-f_{n-1}-f_{a}\right) \mathrm{d} f_{1} \ldots \mathrm{d} f_{n-1}}{\delta\left(f_{1}-f_{a}\right) \ldots \delta\left(f-f_{n-1}-f_{a}\right)}
$$

\subsubsection{Excitation en sinus}

$$
\begin{array}{r}
x(t)=a \cos \left(2 \pi f_{a} t\right) \\
X(f)=a \delta\left(f-f_{a}\right)
\end{array}
$$

Portant l'équation (9) dans (6) on a :

$$
\text { voir équation (10) au-dessus }
$$

$$
\Lambda_{n}\left(f_{a}, \ldots, f_{a}\right)=H_{n}\left(f_{a}, \ldots, f_{a}\right)
$$

Cela revient à dire, en remplaçant dans l'équation (11) $f_{a} \operatorname{par} f$ :

$$
\Lambda_{n}(f, \ldots, f)=H_{n}(f, \ldots, f)
$$

que l'on obtient la fonction de transfert seulement le long de la multisectrice où toutes les fréquences sont égales $f_{1}=f_{2}=\ldots=f_{n}=f$.

Cette information partielle sur $H_{n}$ appelle des excitations multi-harmoniques synchronisées qui complètent les informations sur cette fonction de transfert en dehors de la multisectrice.

\subsection{Calcul des fonctions de transfert par évaluation préalable des multi-correlations dans le domaine temporel}

Afin d'alléger l'écriture, nous présenterons seulement les moments statistiques jusqu'au quatrième ordre. Le moment du troisième ordre $\left(R_{y_{2} x x}\right)$ appliqué à la composante d'ordre 2 de la réponse $y_{2}(t)$ donne; $\tau_{1}, \tau_{2}$ étant les retards temporels [5], $E$ désignant l'espérance mathématique :

$$
R_{y_{2} x x}\left(\tau_{1}, \tau_{2}\right)=E\left[x\left(t-\tau_{1}\right) x\left(t-\tau_{2}\right) y_{2}(t)\right]
$$

avec la définition de $y_{2}$ donnée dans l'équation (1)

$$
\begin{aligned}
& R_{y_{2} x x}\left(\tau_{1}, \tau_{2}\right)= \\
& \quad 2 \int_{-\infty}^{+\infty} \int_{-\infty}^{+\infty} h_{2}(\alpha, \beta) R_{x x}\left(\tau_{1}-\alpha\right) R_{x x}\left(\tau_{2}-\beta\right) \mathrm{d} \alpha \mathrm{d} \beta
\end{aligned}
$$

avec

$$
R_{x x}\left(\tau_{1}\right)=E\left[x\left(t-\tau_{1}\right) x(t)\right]
$$

Par double transformation de Fourier appliquée à l'équation (14) on obtient :

$$
S_{y_{2} x x}\left(f_{1}, f_{2}\right)=H_{2}\left(f_{1}, f_{2}\right) S_{x x}\left(f_{1}\right) S_{x x}\left(f_{2}\right)
$$

avec $S_{x x}(f)=X(f) X^{*}(f), X^{*}(f)$ quantité conjuguée de $X(f)$.

Ainsi, si l'on connaît le premier membre de l'équation (16), il est possible d'attendre la vraie fonction de transfert $H_{2}$ :

$$
H_{2}\left(f_{1}, f_{2}\right)=\frac{S_{y_{2} x x}\left(f_{1}, f_{2}\right)}{S_{x x}\left(f_{1}\right) S_{x x}\left(f_{2}\right)}
$$

L'équation (17) est à comparer à la réponse fréquentielle dans l'équation (5) pour $n=2$

$$
\Lambda_{2}\left(f_{1}, f_{2}\right)=\frac{S_{y_{2} x x}^{\prime}\left(f_{1}, f_{2}\right)}{S_{x x}\left(f_{1}\right) S_{x x}\left(f_{2}\right)}
$$

Dans l'équation (18) $S_{y_{2} x x}^{\prime}$ l'interspectre du troisième ordre est obtenu par une transformation de Fourier à une dimension avec :

$$
S_{y_{2} x x}^{\prime}\left(f_{1}, f_{2}\right)=Y_{2}\left(f=f_{1}+f_{2}\right) \bullet X\left(f_{1}\right) X\left(f_{2}\right)
$$

On peut montrer d'une manière similaire le calcul de l'interspectre d'ordre quatre $S_{y 3 x x x}$ par le biais du moment du quatrième ordre $R_{y 3 x x x}\left(\tau_{1}, \tau_{2}, \tau_{3}\right)$

$$
\Lambda_{3}\left(f_{1}, f_{2}, f_{3}\right)=\frac{S_{y 3 x x x}^{\prime}\left(f_{1}, f_{2}, f_{3}\right)}{S_{x x}\left(f_{1}\right) S_{x x}\left(f_{2}\right) S_{x x}\left(f_{3}\right)}
$$

nous pensons que les fonctions de transfert dans les équations (17) et (20) sont obtenues seulement après avoir évalué les multicorrélations dans le domaine temporel $R_{y 2 x x}, R_{y 3 x x x}$. Ces calculs, certes exigent de nombreuses opérations arithmétiques et une ressource mémoire conséquente. Les progrès accomplis en traitement du signal par des algorithmes rapides [6], d'une part et la nouvelle génération d'ordinateurs d'autre part, permettent de tels calculs directs.

\subsection{Excitation par chocs isolés ou répétés}

Les travaux de Chouychai et Vinh [7] et Tawfiq [2] ont montré que les essais aux chocs permettent d'accéder aux noyaux de Volterra $h_{n}\left(t_{1}, t_{2}, \ldots, t_{n}\right) ; n=1,2,3 \ldots$ L'intérêt de ce type d'essais est que la déconvolution de la série de Volterra est immédiate pourvu que l'on adopte des programmes d'essais appropriés.

Comme exemple, nous présentons des idées directrices pour évaluer les trois premiers noyaux $h_{1}\left(t_{1}\right), h_{2}\left(t_{1}, t_{2}\right)$, $h_{3}\left(t_{1}, t_{2}, t_{3}\right)$ dans l'équation (1).

\section{a) Chocs isolés}

Si l'excitation $x(t)$ est approchée par une fonction impulsion de Dirac

$$
X(t)=a \delta(t)
$$




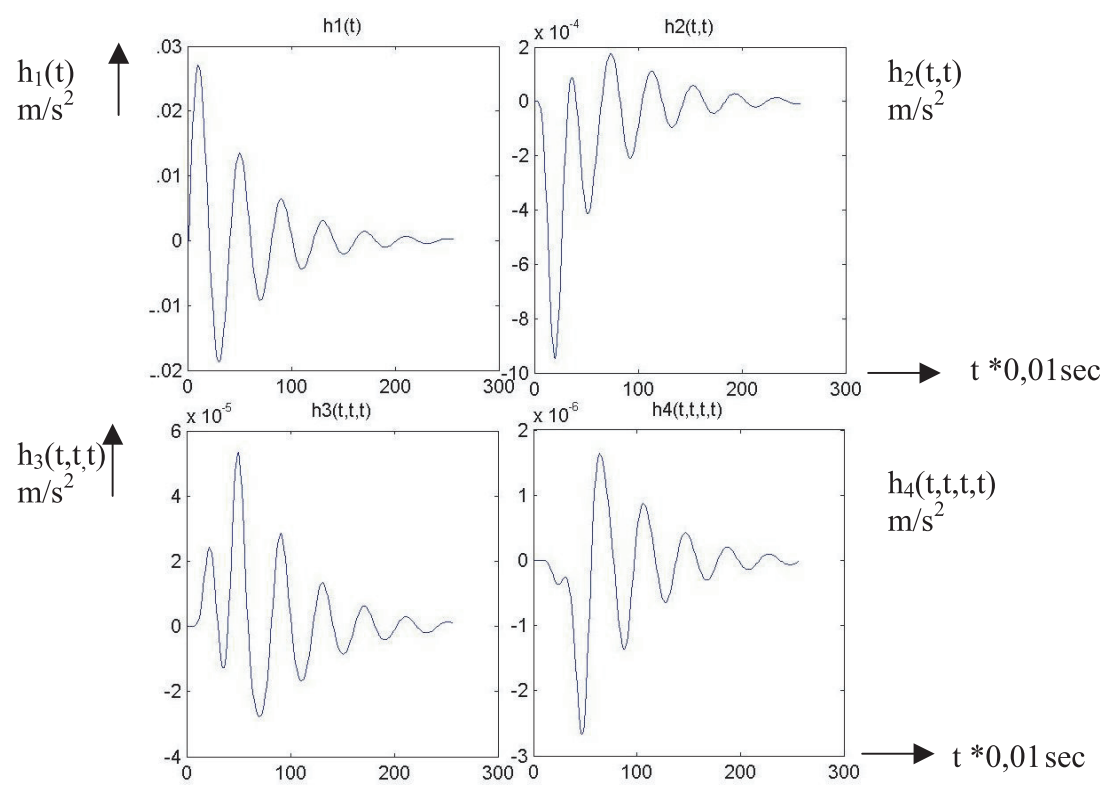

Fig. 1. La réponse impulsionnelle (noyau) d'ordre quatre obtenue à partir de quatre excitations différentes, simulation d'un système non-linéaire $(1 \mathrm{ddl})$ à rigidité quadratique.

Portant l'équation (21) dans (1) et nous limitant aux premiers termes de la série, avec des variables temps égales $t_{1}=t_{2}=t_{3}=t$ :

$$
y(t)=a h_{1}(t)+a^{2} h_{2}(t, t)+a^{3} h_{3}(t, t, t)+O\left[h_{4}\right]
$$

Pour évaluer les noyaux $h_{i}(t, \ldots, t)$ on adopte divers niveaux d'excitation $a_{1}, \ldots, a_{n}$. On écrit donc sous forme matricielle

$$
\{y\}=[a]\{h\}
$$

avec $\{y\}=\left\{y^{(1)} y^{(2)} \ldots y^{(n)}\right\}^{T},\{h\}=\left\{h_{1}(t), h_{2}(t, t), \ldots\right.$, $\left.h_{n}(t, t, t)\right\}^{T}$

$$
[a]=\left[\begin{array}{ccc}
a_{1} & a_{1}^{2} & a_{1}^{n} \\
\cdots & \ldots & \cdots \\
a_{n} & a_{n}^{2} & a_{n}^{n}
\end{array}\right]
$$

On obtient les fonctions noyaux en résolvant l'équation (23).

La simulation de la réponse impulsionnelle d'ordre 4 obtenue avec quatre excitations à différentes amplitudes est présentée en figure 1.

\section{b) Deux chocs décalés dans le temps}

$T$ étant un retard ajustable

$$
x(t)=a \delta(t)+b \delta(t-T)
$$

Nous obtenons, en nous limitant dans (22) à 3 termes pour la réponse

$$
\begin{aligned}
y(t)= & {\left[a h_{1}(t)+b h_{1}(t-T)\right] } \\
+ & {\left[a^{2} h_{2}(t, t)+b^{2} h_{2}(t-T, t-T)+2 a b h_{2}(t, t-T)\right] } \\
& +\left[a^{3} h_{3}(t, t, t)+b^{3} h_{3}(t-T, t-T, t-T)\right. \\
+ & \left.3 a^{2} b h_{3}(t, t, t-T)+3 a b^{2} h_{3}(t, t-T, t-T)\right]
\end{aligned}
$$

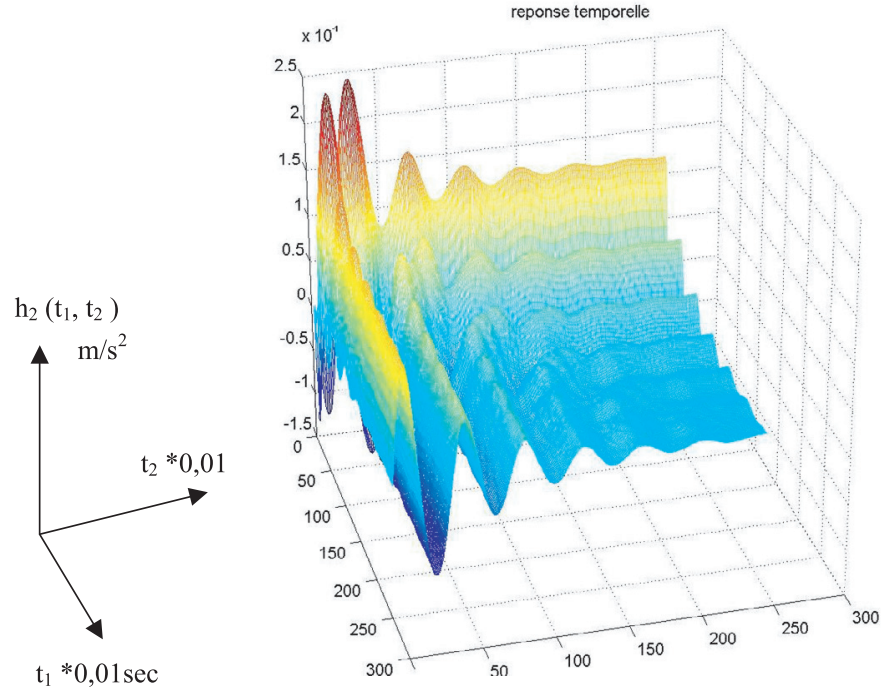

Fig. 2. La réponse impulsionnelle du deuxième ordre du système ci-dessus.

Le second crochet du second membre de l'équation (26) concerne le noyau d'ordre 2 pour des temps égaux et pour des variables de temps différents $(t, t-T)[5]$. Figure 2 représente la réponse impulsionnelle d'ordre 2 (ou le noyau de deuxième ordre) pour un système à rigidité quadratique obtenue avec deux chocs décalé dans le temps (balayage temporel).

Pour le noyau d'ordre 3 , l'information sur $h_{3}$ est partielle notamment pour deux temps égaux, le troisième étant variable. On devra compléter l'information sur $h_{3}\left(t_{1}, t_{2}, t_{3}\right)$ par l'adoption de trois chocs décalés. $T_{1}, T_{2}$ étant des retards ajustables

$$
x(t)=a \delta(t)+b \delta\left(t-T_{1}\right)+c \delta\left(t-T_{2}\right)
$$


Portant l'équation (27) dans (1) on obtient

$$
\begin{aligned}
& y(t)=\left[a h_{1}(t)+b h_{1}\left(t-T_{1}\right)+c h_{1}\left(t-T_{2}\right)\right] \\
& +\left[a^{2} h_{2}(t, t)+b^{2} h_{2}\left(t-T_{1}, t-T_{1}\right)+c^{2} h_{2}\left(t-T_{2}, t-T_{2}\right)\right. \\
& +2 a b h_{2}\left(t, t-T_{1}\right)+2 a c h_{2}\left(t, t-T_{2}\right) \\
& \left.+2 b c h_{2}\left(t-T_{1}, t-T_{2}\right)\right]+\left[a^{3} h_{3}(t, t, t)\right. \\
& +b^{3} h_{3}\left(t-T_{1}, t-T_{1}, t-T_{1}\right) \\
& +c^{3} h_{3}\left(t-T_{2}, t-T_{2}, t-T_{2}\right) \\
& +3 a b h_{3}\left(t, t, t-T_{1}\right)+3 a^{2} c h_{3}\left(t, t, t-T_{2}\right) \\
& +3 b^{2} c h_{3}\left(t-T_{1}, t-T_{1}, t-T_{2}\right) \\
& +3 b^{2} a h_{3}\left(t-T_{1}, t-T_{1}, t\right)+3 c^{2} a h_{3}\left(t-T_{2}, t-T_{2}, t\right) \\
& +3 c^{2} b h_{3}\left(t-T_{2}, t-T_{2}, t-T_{1}\right) \\
& \left.+6 a^{2} b c h_{3}\left(t, t-T_{1}, t-T_{2}\right)\right]+0\left[h_{4}\right]
\end{aligned}
$$

Dans l'équation (28) le seul terme à déterminer est $h_{3}\left(t, t-T_{1}, t-T_{2}\right)$. Le terme de $h_{1}$ est obtenu à partir d'un essai pour une variable de temps, les termes de $h_{2}$ et $h_{3}$ (non soulignés) sont obtenus à partir d'essais pour deux variables de temps, de l'équation (26). À partir de ces termes on extrait le terme de $h_{3}$ (soulignés) en supposant que les termes en $h_{4}$ sont négligeables.

\section{Présentation succincte de l'instrumentation par choc}

Dans [5] nous avons présenté les détails des dispositifs expérimentaux. L'instrumentation préconisée réside dans la mise au point d'une excitation par chocs programmée qui permet d'obtenir les chocs successifs en un ou en plusieurs endroits (entrées multiples) dont les forces mises en jeu sont calibrées en amplitude. Par ailleurs les durées séparant les chocs sont ajustables à l'aide d'un programme informatique, figure 3.

L'intérêt des chocs mécaniques programmés réside dans le contrôle précis des directions des forces appliquées et de la constance de ces dernières. L'utilisation de tels dispositifs nous permet de dire que l'excitation par chocs est aussi fiable que les excitations sinus ou par bruit blanc. La cohérence des fonctions de transfert obtenue est excellente.

Nous attirons l'attention du lecteur sur le fait que l'excitation programmée par chocs répétés reste, jusqu'à plus ample information, la seule méthode expérimentale possible permettant d'évaluer les réponses impulsionnelles des structures non-linéaires.

\section{Système non-linéaire à entrées multiples et sorties multiples (EMSM)}

Les structures de grandes dimensions et/ou complexes telles que les avions ou les voitures automobiles sont considérées comme des systèmes EMSM, déjà dans l'analyse modale classique dite analyse à polyréférences. Pour

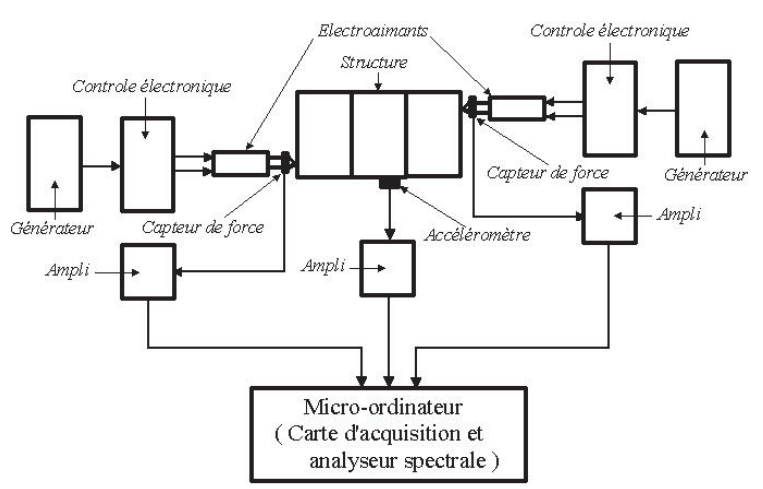

Fig. 3. Dispositif expérimental d'excitation par chocs multiples programmés.

le comportement non-linéaire de telles structures, il faut généraliser la série de Volterra (1) soient $\alpha_{i}(\tau)$. Soit la station $p$ où l'on recueille la réponse ${ }_{p} y(t)$ on écrit donc la série de Volterra généralisée sous la forme

$$
\begin{aligned}
{ }_{p}^{\alpha_{1}, \ldots, \alpha_{q}} y(t) & ={ }_{p} y_{0}+\sum_{i=1}^{q} \int_{-\infty}^{+\infty}{ }_{p}^{\alpha_{i}} h_{1}\left(\tau_{1}\right) x_{i}\left(t-\tau_{1}\right) \mathrm{d} \tau_{1} \\
+ & \sum_{i=1}^{q} \sum_{j=1}^{q} \int_{-\infty}^{+\infty} \int_{-\infty}^{+\infty}{ }_{p}^{\alpha_{i} \alpha_{j}} h_{2}\left(\tau_{1}, \tau_{2}\right) \\
& \times x_{i}\left(t-\tau_{1}\right) x_{j}\left(t-\tau_{2}\right) \mathrm{d} \tau_{1} \mathrm{~d} \tau_{2} \\
+ & \sum_{i=1}^{q} \sum_{j=1}^{q} \sum_{k=1}^{q} \int_{-\infty}^{+\infty} \int_{-\infty}^{+\infty} \int_{-\infty}^{+\infty}{ }_{p}^{\alpha_{i} \alpha_{j} \alpha_{k}} h_{3}\left(\tau_{1}, \tau_{2}, \tau_{3}\right) \\
\times & x_{i}\left(t-\tau_{1}\right) x_{j}\left(t-\tau_{2}\right) x_{k}\left(t-\tau_{3}\right) \mathrm{d} \tau_{1} \mathrm{~d} \tau_{2} \mathrm{~d} \tau_{3}
\end{aligned}
$$

Le nombre de noyaux de Volterra du même ordre $n$ s'en trouve augmenté. Pour une sortie unique $p$ on a pour $h_{1}, N q$ termes, $N$ étant le degré de liberté de la structure, $q$ le nombre de stations d'excitation.

Pour les modes propres secondaires on a $(N+M) q^{2}$, $M$ étant le nombre des valeurs propres secondaires obtenues par couplages des pulsations propres primaires.

Pour les modes propres tertiaires on a $(N+M+R) q^{3}$, $R$ étant le nombre des valeurs propres tertiaires obtenues par couplages des pulsations propres primaires et secondaires.

Si l'on choisit plusieurs stations $p$ confondues avec l'ensemble des stations d'excitations, alors il faudra multiplier les nombres de termes présentés plus haut par $q$.

Les termes diagonaux tels que ${ }_{p}^{\alpha_{1}, \ldots, \alpha_{q}} h_{n}\left(t_{1}, \ldots, t_{n}\right)$ avec des pré-exposants égaux constituent les noyaux classiques de Volterra. Pour les pré-exposants $\alpha_{i}, \ldots, \alpha_{q}$ différents dans l'équation (29) correspondant aux noyaux «non-diagonaux », on peut réduire leur nombre par des considérations de symétrie (commutativité des facteurs des produits dans (29)) concernant les stations d'excitations. Par contre le principe de Maxwell qui permet d'intervertir l'excitation et la réponse, applicable pour des systèmes linéaires ne l'est pas ici. On note dans ce sens les travaux de Worden [8]. 


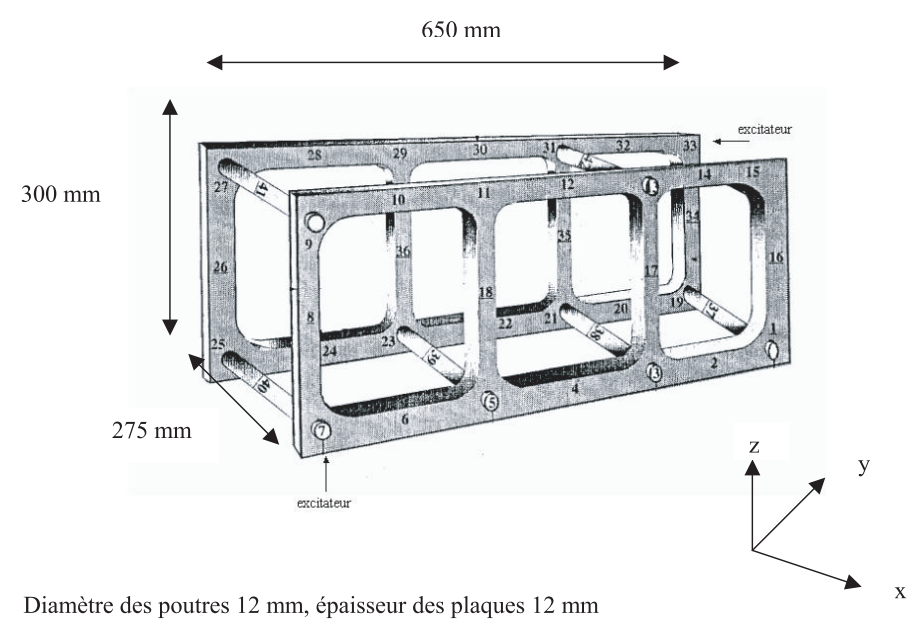

Fig. 4. Une structure en aluminium assemblée, les points indiqués précisent la position de deux excitateurs.
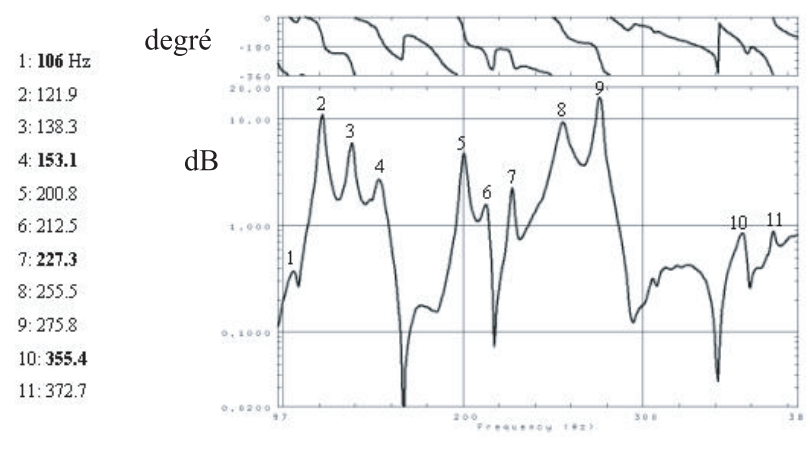

phase

module

$\mathrm{Hz}$

Fig. 5. Une fonction de réponse fréquentielle (inertance) due à deux excitations (par choc) simultanées du berceau. Les chiffres en gras représentent les fréquences obtenues par la mono-excitation, on constate la présence de certaines fréquences supplémentaires, excitations en $-7 x,-33 y$ et la réponse en $12 x$.

La figure 4 montre une maquette utilisée pour le test en multi-excitation (polyréférence). La figure 5 représente la fonction de réponse fréquentielle due à deux excitations par chocs simultanés, on obtient des modes supplémentaires, elle montre la nécessité d'utiliser les séries des fonctionnelles non-linéaires pour une étude approfondie qui serait l'objet d'une publication future.

\section{Conclusion}

1. Les relations entre les fonctions de transfert et les réponses fréquentielles (ou fonctions de transfert dégénérées) ont été présentées et permettent l'exploitation de ces dernières pour obtenir des informations partielles sur les noyaux.

2. La transformée de Fourier unidimensionnelle trouve rapidement ses limites en analyse modale non-linéaire. Il faudra recourir à des calculs directs de multicorrélations dans le domaine temporel.

3. Les essais d'impact présentent un grand intérêt car ils permettent l'accès direct aux fonctions noyaux de Volterra moyennant une instrumentation et une programmation adéquates des essais.

4. La série de Volterra peut être généralisée aux systèmes à entrées et sorties multiples.

\section{Références}

[1] I. Tawfiq, T. Vinh, Extension of modal analysis to nonlinear structures using Volterra functional series, J. Mech. Syst. Signal Processing 17(2) (2003) 379-407

[2] I. Tawfiq, T. Vinh, A new approach to impact testing instrumentation using electronic slave hammer, J. Mech. Syst. Signal Processing 11 (1997) 296-285

[3] S.J. Gifford, Volterra series analysis of non-linear structures, Ph.D. thesis, Heriott Watt University, Edinburgh, March 1989

[4] D. Storer, Dynamic analysis of non-linear structures using higher order frequency response, Ph.D. thesis, University of Manchester, October 1991

[5] I. Tawfiq, T. Vinh, Étude des structures non-linéaires par chocs programmés, $\mathrm{XV}^{\mathrm{e}}$ Congrès Français de Mécanique, $\mathrm{n}^{\circ}$ 424, Nancy, 3-7 septembre 2001

[6] H. Liu, Traitement du signal multidimensionnel et série de Volterra, Thèse de doctorat CNAM Paris, 28 avril 1989

[7] T. Vinh, T. Chouchai, H. Liu, M. Jouder, Second order transfer function, computation and physical interpretation, Proceeding of the 5th IMAC, vol. I, 1987, pp. $587-592$

[8] K. Worden, G. Manson, G.R. Tomlinson, A harmonic probing algorithm for multi input Volterra series, J. Sound and Vibration 201(1) (1997) 67-89 